\title{
ALICE: A non-LTE plasma atomic physics, kinetics and lineshape package
}

\author{
E.G. Hill ${ }^{1}$, G.Pérez-Callejo ${ }^{2}$, and S. J. Rose ${ }^{1,2,{ }^{*}}$ \\ ${ }^{1}$ Plasma Physics Group, The Blackett Laboratory, Imperial College London, Prince \\ Consort Road, London, SW7 2AZ. UK \\ ${ }^{2}$ Department of Physics, Clarendon Laboratory, University of Oxford, Parks Road, Oxford \\ OX1 3PU, UK
}

January 15, 2018

\begin{abstract}
All three parts of an atomic physics, atomic kinetics and lineshape code, ALICE, are described. Examples of the code being used to model the emissivity and opacity of plasmas are discussed and interesting features of the code which build on the existing corpus of models are shown throughout.
\end{abstract}

\section{Introduction}

ALICE is a plasma atomic physics, atomic kinetics and lineshape package: it calculates the energy levels and cross sections for transitions in a given ion which are then used to perform an atomic kinetics calculation to find the populations of those levels, and used to produce the emissivity and opacity of the material. The radiation field produced is fed back self-consistently and spectrally resolved into the atomic kinetics to calculate the trapping of the radiation in a given geometry, and the entire problem is solved time dependently for either a single material or a mixture. ALICE was designed to model experiments on the ORION short pulse laser system at AWE and includes various corrections to account for effects on the energy levels and lineshapes in dense plasmas. Experimental results in the regimes accessed in those experiments will be compared to ALICE in the paper.

Several detailed atomic physics codes for calculating the energy levels in ions and the rates between them have been written, for example the codes at the Los Alamos National Laboratory [1], HULLAC[2], or FAC [3]; and there are also older, less detailed codes using analytic approximations, for example GALAXY [4], and more limited calculations performed in LTE when the full compliment of rates are not required, for example DAVROS [6] or IMP [7]. There are a number of atomic kinetics models in use, often drawing on an atomic physics code (for example [2]) or using the input from such a code, for example FLYCHK [8], SCRAM

\footnotetext{
*Corresponding author: Steve Rose s.rose@imperial.ac.uk
} 
[9] or CRETIN [10]. Lastly many lineshape models exist, both incorporated into code such as FLYCHK, ATOMIC or CRETIN or as standalone codes of varying levels of sophistication [11].

Papers such as [4] or [12] provide a good summary of the operation of such models and show the basic structure of any code performing atomic physics, kinetics and lineshape calculations. Also [1] provides a comprehensive discussion of the rate and energy level calculations involved in producing a detailed term accounting (DTA) model within a consistent framework. In this paper we will therefore focus on the aspects of ALICE which are particularly different from these models or which lead to different results.

The first section covers the atomic physics calculations, discussing the methods used to calculate the energy levels and cross-sections required for the atomic kinetics model. In the second section we discuss the atomic kinetics model, divided into four sub-sections.

1) We begin by describing the overall flow of the program and how time dependence is included, followed by a demonstration of the importance of time dependence in short-pulse laser-plasma interactions.

2) We then discuss the effects of optical depth, which are illustrated by analysis of a K-shell Scandium experiment where two spectrometers at different angles to the target normal must be modelled simultaneously.

3) Completeness of configurations is important in any atomic kinetics model, particularly in the inclusion of satellite lines, autoionising and Rydberg states, and we demonstrate ALICE's ability to deal with such situations in the more computationally complex cases of open L-shell Chlorine and Germanium.

4) Finally, at high densities lineshapes play an important role as an experimental diagnostic - we discuss the lineshape model in ALICE and show how comparison with the same K-shell Scandium results motivates the inclusion of higher order multipole effects in Ly- $\gamma$ and He- $\gamma$ lines.

\section{Atomic physics}

\subsection{Orbitals and basic atomic structure}

The methods used in the atomic physics calculations in ALICE are different to the more usual methods involving Racah algebra. ALICE instead uses a novel approach which directly uses Slater determinants. The details of this method are discussed in $[13,14]$.

The radial wavefunctions for the configurations along with the energies of those configurations are obtained using Cowan's atomic structure code, RCN[15]. This has been modified, firstly to output the required quantities in a format for reading into ALICE, and secondly to give an additional stability when calculating excited neutral atoms which is useful for cold K-shell spectroscopy (involving a modification to introduce a permanent slight damping in the calculation of the radial wavefunctions). RCN[15] is fast, stable, and produces reliable energy differences, even for the exotic configurations found in hot dense plasmas. The file format is designed to be simple so that the code could be easily replaced by other models which calculate wavefunctions in plasma conditions (PURGATORIO [16] for example). The energies returned are those for configurations defined in $n l$ notation, for example $1 s^{2} 2 s^{2} 2 p^{2}$, reflecting the focus of ALICE on low to mid-Z ions. The small part of the wavefunction, $Q(r)$ is not used in ALICE, and the large part is normalised to account for this.

Using these energies and wavefunctions we calculate the Hamiltonian matrix for the fine structure states 
within the configuration and through diagonalisation obtain the eigenvalues (energies) of the states and their eigenvectors which are linear combinations of the determinantal basis states. Our method is based on the observation that we can choose a set of $M$ states (which we represent as linear combinations of Slater determinants) such that only one represents each fine structure state, while still being able to calculate all required matrix elements between those states for the transition matrix element calculations.

The results have been compared to various sources of experimental and theoretical data and are found to be in sufficiently good agreement for $Z \leq 36$ which covers a large number of elements of laboratory and astrophysical interest. The errors in the energy levels for simple configurations e.g. He-like, are typically $\sim$ $0.1 \mathrm{eV}$ for $\mathrm{Al}(Z=13)$ and $\sim 1 \mathrm{eV}$ for $\operatorname{Kr}(Z=36)$. The term structure including and excluding configuration interaction has been extensively tested both against other theoretical data, tabulated experimental data and by comparing to experimental data through the use of the atomic kinetics model. Typical agreement is shown in Figure 1 - full configuration interaction is included and since the total spread of the levels is 13.7 Ryd, the energy deviations of ALICE are all $<0.3 \%$ relative to that spread. Configuration interaction amongst sets of levels can be included when the levels to be calculated are selected. Spin-orbit interaction, sometimes called limited configuration interaction, is always included.

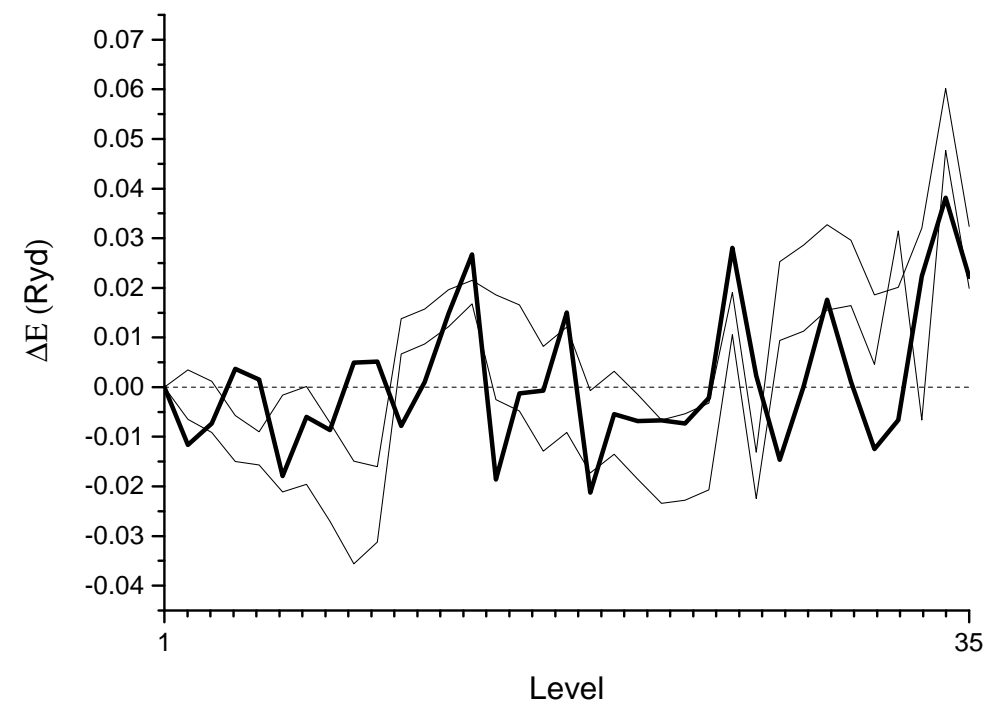

Figure 1: A comparison of the deviations from GRASP[17] of ALICE (bold) and SS[18] and CAHF[19] using data from [20]. We consider the energy levels in the configuration $1 s^{2} 2 s^{2} 2 p^{6}\left\{3 s^{2}, 3 s 3 p, 3 s 3 d, 3 p^{2}, 3 p 3 d, 3 d^{2}\right\}$ in Fe XV $(Z=26)$, where the \{\} denote configuration interaction between the levels. The lines are to guide the eye, and the dashed line denotes zero deviation. The energies in all cases are relative to the lowest energy level. The abscissa refers to the labelling of the levels in [20]. 


\subsection{Calculation of the collisional cross sections}

In the calculation of collisional cross-sections, the main problem is the calculation of the radial integrals, and in particular reaching convergence of the sum over angular momenta of the free electrons in the dipole transition case. The minimisation of the number of radial integrals which must be calculated has been the subject of considerable study ([1] Section 4) involving the truncation of the sum of angular momenta as early as possible and the calculation of the cross-section at the smallest possible number of energies to minimise the number of such integrations consistent with a sufficiently accurate result.

ALICE uses a parametrisation of the collisional excitation cross-section which is different to that usually used, and which is related to the method of minimising the number of radial integrals. ALICE lies between the two approaches of either calculating many points of the cross-section and then performing a least-squares fit to those points while in some cases trying to maintain some physical limits, and calculating a simple crosssection and then applying scalings to it in order to reproduce behaviour seen in other calculations[23, 24]. In particular we wish to avoid calculating distorted wave collision strengths, particularly those for the dipole transitions and particularly at energies significantly above threshold. This is because at those energies, as well as an increase in the difficulty of the numerical calculation due to the highly oscillatory nature of the functions [24], the number of partial waves which must be summed becomes very large.

The resulting fits aim for an accuracy somewhere between the large scale calculations with large numbers of distorted waves[1] and those which employ techniques such as scaling the cross-sections obtained from the Plane Wave Born (PWB) approximation[23, 25]. The fits in ALICE include more physics than the latter, for example the exchange contributions, with a minimum amount of extra numerical effort. Accuracies for the scaled PWB calculations are typically $20 \%[24,15]$ or $30 \%[25]$ near threshold, and they have no ability to be accurate for exchange dominated transitions since exchange cross-sections cannot be calculated using this method. The accuracy of the distorted wave calculations[1] is hard to define; the agreement between different codes is generally good $(\sim 5-10 \%)$ and the agreement of those codes with experiment [26] is at the $10 \%$ level. We are therefore aiming for fits at the $5-10 \%$ level to bring the results of our code into line with other more detailed codes.

The fit used in ALICE is

$$
\sigma(E) E=\left[c_{0}+c_{3} \ln (U)\right] g(U)+(1-g(U))\left[c_{2}+c_{1} \ln (U)\right]+c U^{a}\left(\frac{d}{d+U}\right)^{-2.5-a}
$$

where

$$
g(U)=\frac{A}{(U-1)+A}
$$

where $E$ is the incident electron energy, and $\Delta E$ is the energy change of the transition. In the case of direct transitions (those with $r=0^{*}$ ), the rates at high energies are calculated using the Plane Wave Born approximation and generalised oscillator strengths [15]. This, since $g(U) \rightarrow 0$ as $U=E / \Delta E \rightarrow \infty$ allows us to fit the values of $c_{2}$ and $c_{1}$, therefore obtaining a form consistent with the Bethe high energy limit (a feature we will return to below). For $t=1^{*}, c_{2}$ and $c_{1}$ are both non-zero, whereas if $t \neq 1^{*}$, we assume $c_{1}=0$. Near threshold, the cross section deviates from the PWB form - here the details of the interaction between

\footnotetext{
* $\mathrm{t}$ and $\mathrm{r}$ are the rank of the associated tensor operator in the orbital and spin angular momentum transition matrix elements respectively $[13,14]$
} 
the continuum and bound electrons are important and hence we cannot expect to have a good accuracy without performing some kind of calculation including the ion structure. Two distorted wave calculations are performed at energies $\Delta E+\epsilon$ and $\Delta E+2 \epsilon$, close to threshold, and determine the values of $c_{0}$ and $c_{3}$. The value of $A$ in $g(U)$ is fixed for different types of transition and is the characteristic value of $U$ where the PWB approximation breaks down. The cross-section is relatively independent of $A \sim 1$, however we find that $A=1 / 2$ for direct dipole matrix elements and $A=3 / 2$ for direct non-dipole elements gives good fits to other results.

We next consider the indirect part of the transition, $r=1^{*}$, which we fit using the final term in the cross-section expression, which allows an asymptotically appropriate form with a deviation near $U=1$. The indirect and exchange terms converge very quickly with few continuum electron angular momenta.

Some examples of the cross-sections calculated using this method are shown in Figure 2:

Firstly we consider the $1 s 2 s{ }^{1} S_{0} \rightarrow 1 s 2 p{ }^{1} P_{1}$ transition in Aluminium in Figure 2a. The graph shows the results from the LANL suite of codes (black) with deviations from that result of $\pm 5 \%$ (black dashed) along with the result from ALICE (red) and from FAC (blue). The agreement between the codes is generally good. The cross-sections have also been compared for Krypton and the decrease in discrepancy near threshold supports the assertion that these differences are due to the form of the bound-continuum electron interaction. The discrepancy is similar to that of the results of HULLAC ([2] Figure 4). ALICE gives a slightly different asymptotic form of the cross-section at high energies when compared to the LANL codes, which appears to be attributable to the difference in the dipole matrix element between the states, and therefore to the underlying atomic physics. Taking the common, strong $1 s^{2}{ }^{1} S_{0} \rightarrow 1 s 2 p{ }^{1} P_{1}$ transition in Aluminium as an example, oscillator strength values in the literature range from $f=0.742[27]$, through FAC's $f=0.768$ and ALICE's value of $f=0.777$ to the LANL value of $f=0.808$. These differences affect the calculation because of the relationship between the dipole strength and the Bethe high energy asymptote (e.g. [28]); $c_{1}$ is proportional to the dipole oscillator strength.

Secondly, we look at the intercombination transition, $1 s^{2}{ }^{1} S_{0} \rightarrow 1 s 2 p{ }^{3} P_{1}$. Figure $2 \mathrm{~b}$ shows the fit, where $A=1 / 2, c_{0}=2.19 \times 10^{-21}, c_{1}=5.41 \times 10^{-21}, c_{2}=1.04 \times 10^{-21}, c_{3}=2.96 \times 10^{-23}, c=2.67 \times 10^{-19}$, $d=19.03$ and $a=1.8$ (where $\sigma(E) E$ is in units of $\mathrm{cm}^{2} R y d$ ). It is interesting that the threshold value of the cross section for the $1 s^{2}{ }^{1} S_{0} \rightarrow 1 s 2 p{ }^{1} P_{1}$ transition is $\sim 9 \times 10^{-19} \mathrm{~cm}^{2} R y d$ and therefore for temperatures around $300-500 \mathrm{eV}$ where He-like Aluminium is most prevalent at solid densities, the collision strength from the ground state $1 s^{2}{ }^{1} S_{0}$ to the $1 s 2{ }^{3} P_{1}$ level is about 2 orders of magnitude larger than would be expected from the ratio of dipole strengths. This could not have been predicted from the PWB collision strengths.

\subsection{Relativistic formulae}

Because of the use of an exact high energy limit with the coefficients $c_{1}$ and $c_{2}$, the formulae shown above extend to the relativistic case.

For the dipole allowed transitions, including relativistic effects on the free electron, it is necessary to modify equation 1 , by replacing

$$
\frac{1}{E}\left(c_{1} \ln U+c_{2}\right) \rightarrow \frac{2 R y d}{m v^{2}}\left(c_{1}\left[\ln \left(\frac{\beta^{2}}{1-\beta^{2}}\right)-\beta^{2}\right]+\left[c_{1} \ln \left(m c^{2} / 2 R y d\right)+c_{2}-c_{1} \ln \Delta E\right]\right) .
$$

where Ryd stands for a Rydberg unit of energy $(\sim 13.605 \mathrm{eV})$, included in the equation since $E$ and $\Delta E$ are 


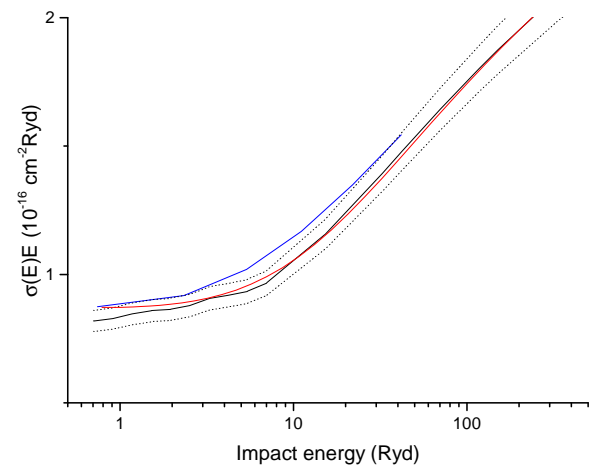

(a)

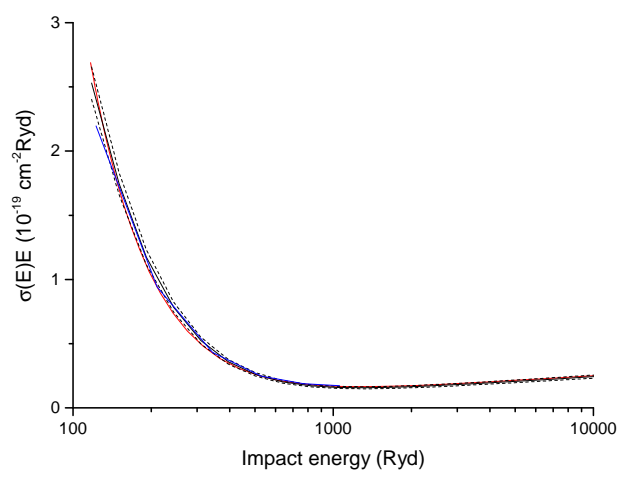

(b)

Figure 2: Examples of the fitting of (2a) a direct, dipole transition, and (2b) a transition with a significant indirect component. The transitions are in He-like Al, and the results from ALICE (red) are compared with those from FAC (blue) and the LANL suite of codes (black).

$\begin{array}{llll}\text { Energy }(\mathrm{keV}) & \text { Bostock and Fontes[29] } & \text { ALICE (NR) } & \text { ALICE (R) } \\ 50 & 93 & 91 & 95 \\ 100 & 68 & 60 & 71 \\ 500 & 43 & 19 & 46 \\ 1000 & 44 & 11 & 47 \\ 5000 & 57 & 3 & 64\end{array}$

Table 1: We compare cross sections from ALICE against those reported in [29]. The cross sections are for $\mathrm{Z}=281 s^{1 / 2} \rightarrow 2 p^{3 / 2}$ electron-impact excitations. The energy is that of the incident electron and the cross sections are in barns. (NR) are non-relativistic and (R) are relativistic as discussed in the text.

measured in Rydbergs.

For direct, but dipole disallowed transitions, the modification in equation 1 is

$$
\frac{1}{E} c_{2} \rightarrow \frac{2 R y d}{m v^{2}} c_{2} .
$$

Other parts of the cross-section are unimportant at relativistic energies for elements we consider here, and more complex implementation would only be consistent with a relativistic level of treatment of the atomic physics and of the free electron-ion interaction which are not present in ALICE, see [29]. In Table 1 we compare the cross sections derived in this way with those from [29] for the $1 s_{1 / 2} \rightarrow 2 p_{3 / 2}$ transition in $\mathrm{H}$-like Ni. We see that the increase (rather than decrease) of the cross section with energy at high energies when relativistic effects are included, commonly called the 'relativistic rise', is reproduced.

The calculation of relativistic cross sections is very important for including the effect of 'hot' or 'fast' electrons which have typical energies around $1 \mathrm{MeV}$ which are produced in the interaction of high powered 
lasers with both gas and solid targets. As shown in Table 1, a $5 \mathrm{MeV}$ electron energy which is typical in laser-plasma interactions at laser intensities of $10^{20} \mathrm{Wcm}^{-2}$ has a cross-section about 20 times larger than the non-relativistic result.

\subsection{Collisional and photo-ionisation rates}

The photoionisation cross sections are calculated in the Distorted Wave Approximation, and are fitted using a $c_{1} U^{-c_{2}}$ form, where values of $c_{2}$ of 2 to 3 are typical[30]. The angular parts are calculated in a very similar way to that for the rates above, and it can be shown [1] that the angular parts required for the photoionisation cross sections are identical to those needed for collisional ionisation, despite all multipole elements needing to be considered in the latter case. The factoring out of the angular part of the matrix elements renders the collisional ionisation cross-section between pure J-states separable into angular and radial parts, and so the latter can be evaluated separately. The energy dependence of the collisional ionisation cross-section is highly independent of the atomic structure, while also being very computationally expensive to calculate (requiring a sum over two degrees of freedom in the free electron energies). We use the tabulations of the appropriate energy-summed radial integrals found in [1].

\subsection{Autoionisation rates}

These are calculated in ALICE by decoupling the 4 active electrons (two from the initial state, one from the final state and one from the continuum) obtaining a double sum over fractional parentage coefficients, and a number of $6 \mathrm{j}$ and $9 \mathrm{j}$-symbols. These matrix elements have been validated against those from FAC, and, although the values differ slightly due to the extreme sensitivity to the radial matrix elements, an overall linear correlation between the values can be seen as is shown in Figure 3, where the autoionisation rates in Aluminium XII (He-like) from the $\left\{2 s^{2}, 2 s 2 p, 2 p^{2}\right\} \rightarrow 1 s$, Aluminium XI (Li-like) $1 s\left\{2 s^{2}, 2 s 2 p, 2 p^{2}\right\} \rightarrow 1 s^{2}$ and Aluminium X (Be-like) $1 s\left\{2 s^{2} 2 p, 2 s 2 p^{2}, 2 p^{3}\right\} \rightarrow 1 s^{2}\left\{2 s^{2}, 2 s 2 p, 2 p^{2}\right\}$ are shown, along with a diagonal dashed line which the points would lie on if the results from FAC and ALICE were equal. This sensitivity to these elements will become increasingly important in atomic kinetics calculations at low densities - ensuring the completeness of autoionising configurations and the accuracy of the autoionisation rates continues to be a challenge for atomic kinetics codes.

\subsection{Parallelisability}

For a typical calculation using thousands or tens of thousands of levels, hundreds of CPU hours of computational time are required, and hence it is desirable to have a code which parallelises efficiently. Due to the nature of these calculations, particularly the independence of the different cross section calculations, we expect to be able to achieve close to a linear scaling with the number of cores. Tests have been performed on the cx1 computer at Imperial College [31] with up to 72 cores, and a linear scaling is found. Due to the large amount of reading and writing from and to files which is required in ALICE, this scaling may be expected not to hold on bandwidth limited computing systems, and there are also small deviations from a linear scaling when the total number of cores is kept constant and the number of cores used per CPU is varied. 


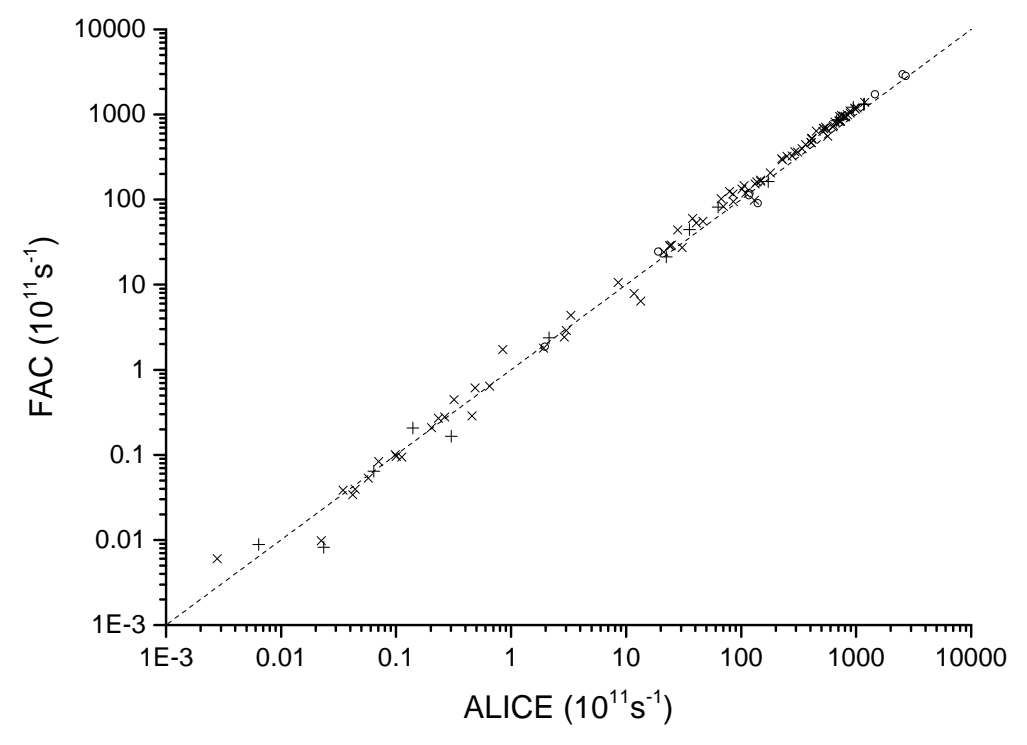

Figure 3: Autoionisation rates in ALICE for the Aluminium XII (He-like) $\left\{2 s^{2}, 2 s 2 p, 2 p^{2}\right\} \rightarrow 1 s$, Aluminium $\mathrm{XI}$ (Li-like) $1 s\left\{2 s^{2}, 2 s 2 p, 2 p^{2}\right\} \rightarrow 1 s^{2}$ and Aluminium X (Be-like) $1 s\left\{2 s^{2} 2 p, 2 s 2 p^{2}, 2 p^{3}\right\} \rightarrow 1 s^{2}\left\{2 s^{2}, 2 s 2 p, 2 p^{2}\right\}$ transitions are compared against those from FAC. The diagonal dashed line is the line the points would lie on if the results from ALICE and FAC were equal.

\subsection{The DCA-type model}

ALICE also allows the construction of a DCA-type model, where the levels in a configuration are averaged together in the calculation of the rates. The photoexcitation rates are excluded from this averaging and this allows the photoexcitation rates and, importantly, the emission, to always be treated in a DLA model. UTAs are not used; however the assumption of Boltzmann statistics among the fine structure levels is made. No correction is made for this and the assumption of statistical distribution between the levels within a configuration is noted as a shortcoming of the approximation. The conditions which ALICE is designed to work in (high temperature and density plasmas) are those in which this approximation would be expected to hold well, and, in the future, corrections involving an effective temperature could be implemented. The rates other than photoexcitation rates for the averaged levels are calculated simply using analytic formulae for rates between averaged levels [1], and therefore ignore the populations of the incident levels which are assumed to be proportional to their degeneracy. This is inaccurate both when in LTE (by a Boltzmann factor) or when out of LTE, and is recognised as a deficiency of the model, particularly in low density, non-LTE plasmas.

This model can be used for LTE calculations at any density; the use of detailed dipole-allowed transition arrays allows the porosity of those arrays to be treated correctly at low densities unlike in the UTA 
approximation leading to more accurate spectra in that regime.

In order to decrease computational time, ALICE allows for a mixture of the DCA and DTA descriptions. For example, in the experiment considered below involving K-shell Scandium the set of levels employed is extended down to Ne-like in order to remove any dependence on the initial conditions. The DTA model is only used for the diagnostically important Be-, Li-, He- and H-like levels. This results in a considerable decrease in computational time while also allowing a satisfactory completeness of configurations to be obtained. However, if for example, Ne- and Na-like Scandium were to be investigated, a new model using a DTA description in the region of those charge states and a more averaged DCA description elsewhere would be created.

For all the DTA rates discussed in the previous four sections, tests have been undertaken, involving checking that the rates between configurations are the same when evaluated numerically from the average of DTA rates and when calculated from the formulae (see e.g. [1]) found by performing this average analytically. The analytic formulae contain no factors relating to the angular momenta of the electrons, leading to very simple formulae which are easy to implement.

\section{$3 \quad$ Atomic kinetics}

In this section we describe the atomic kinetics model. ALICE was developed to provide a new, independent and robust model for diagnosis of the density and temperature of high density, high temperature (solid density, hundreds of eV) plasmas such as are created in buried layer targets heated by the ORION laser at AWE, Aldermaston. These experiments aim to use a mixture of low and high $\mathrm{Z}$ materials to allow the opacity of the high $\mathrm{Z}$ materials to be determined at a temperature and density which is well characterised. To analyse these experiments ALICE must address the following considerations:

1) The timescales for the heating and hydrodynamic expansion of these targets is of the same order as the ionisation timescales of the plasma and therefore the ionisation states of the mixture of materials must be solved self-consistently and time dependently.

2) The spatial dimensions of the layers (typically $\sim 0.1 \mu \mathrm{m}$ ) result in the strong transition lines having significant optical depth. This has an effect on the level populations and radiation leaving the plasma. In the conditions of temeprature and density encountered in the experiments, existing methods based on escape factors would be expected to be unreliable because of the departure of the lineshapes from Doppler, Lorentzian or Voigt. Instead we employ a radiation transport calculation with accurately calculated lineshapes coupled in a frequency resolved way to the atomic kinetics.

3) Mixtures of low, mid and high $\mathrm{Z}$ materials must be treated, requiring a self-consistent description of each material and a dependence of the lineshapes and pressure ionisation on the properties of the surrounding mixed-material environment.

The code has three different levels of detail - full DTA, a DCA model with detailed line positions (the atomic data production for these two was described above), and an effective-temperature modified ThomasFermi model. These are intended to be used for low, mid and high $\mathrm{Z}$ materials respectively. 


\subsection{The scheme of the code}

The scheme may be written in pseudo-code as:

1. Read in the atomic physics data for the elements and initialise the code

2. Solve for steady state at the initial temperature and material density

2.1 Guess the initial $n_{e}$ and radiation field

2.2 For each element

2.2.1 Calculate the rate matrix for current guesses of $n_{e}$, radiation field, $T_{e}, T_{i}, \ldots$ obtaining the spectrum

2.2.2 Solve for the populations using the current value of $n_{e}$, to obtain the charge state

2.3 Using the charge states and spectra found for the elements, recalculate the values of $n_{e}$ and the total radiation field, and loop to 2.2 until consistency is reached

\section{Move forward one time step}

3.1 Use the current charge states and radiation field as an initial guess, along with density and $T_{e}$ from the time history

3.2 For each element

3.2.1 Calculate the rate matrix for current guesses of $n_{e}$, radiation field, $T_{e}, T_{i}$, and populations

3.2.2 Solve implicitly for the populations using the current values, to obtain the charge state

3.2.2.1 Loop to 3.2.2 iterating only on the charge state for that single element

3.3 Using the charge states and spectra found for the elements, recalculate the values of $n_{e}$ and the total radiation field, and loop to 3.2 until consistency is reached.

4. Output diagnostic information - charge states and spectra - and loop to 3 until end of time series is reached

In more detail, ALICE solves

$$
\frac{d \mathbf{n}}{d t}=A \mathbf{n}
$$

where $\mathbf{n}$ is the vector of populations of the levels and $A$ is the rate matrix. Due to the poor conditioning of the rate matrix, this equation must be solved implicitly, and due to the dependence of $A$ on the populations $\mathbf{n}$ through both the charge state and through the radiation field it must be solved by iteration to consistency. We define $\mathbf{n}_{i, j}$ as the $j$ th iteration involved in finding the populations at timestep $i$, and

$$
\mathbf{n}_{i+1, \infty}=(I+A \Delta t)^{-1} \mathbf{n}_{i, \infty}
$$

is the equation to be solved, where $I$ is the identity matrix. However $A=A\left(\mathbf{n}, T_{e}, I(\nu), n_{e}, \ldots\right)$, and Step 2 above involves solving the same equation in the limit of large $\Delta t$ to obtain the initial steady-state populations. We note that throughout we assume the material motion to be Lagrangian and non-relativistic, and that the plasma consists of a single, uniform slab of constant composition with a known history of temperature, density and extent.

We begin with $\mathbf{n}_{i, \infty}$, the populations at timestep $i$ after convergence of the iterations for that timestep, and form $A=A\left(\mathbf{n}_{i, \infty}, T_{e(i+1)}, I(\nu)_{i, \infty}, n_{e i, \infty}, \ldots\right)$ as an initial guess for $A$, and then solve for $\mathbf{n}_{i+1,0}$. We then iterate, incrementing $j$, by defining $\mathbf{m}=\beta \mathbf{n}_{i+1, j}+(1-\beta) \mathbf{n}_{i, \infty}$ where $A=A\left(\mathbf{m}, T_{e(i+1)}, I(\nu)_{i+1, j}, n_{e}(\mathbf{m}), \ldots\right)$. ALICE allows the choice of $\beta$ between 0 and 1 with $\beta=1$ being fully implicit and $\beta=0.5$ being usually used. 
We solve for $\mathbf{n}_{i+1, j+1}$ using this $A$ in Equation 6, and thereby obtain $I(\nu)_{i+1, j+1}$. To stabilise the iterations, we then replace all the $x_{i+1, j+1}$ quantities with suitably damped and limited values. For example $I(\nu)_{i+1, j+1}$ is calculated as $\alpha I(\nu)_{i+1, j+1}+(1-\alpha) I(\nu)_{i+1, j}$ and then bounded below by $0 . \mathbf{n}_{i+1, j+1}$ is similarly damped, bounded below by 0 and renormalised.

$\alpha$ is a damping factor which is chosen in ALICE to achieve a balance between stability and computational time. Unlike $\beta$, where the values considered are taken at the previous and current timesteps, $\alpha$ relates to consecutive iterations of a value at the current timestep. Both of these factors can be used at the same time, the value found at the current iteration being damped (with $\alpha$ ) followed by using a chosen $\beta$ to determine how that variable is used in the calculation of $A$. Explicitly, we first calculate $\mathbf{n}_{i+1, j}$ and then damp it against $\mathbf{n}_{i+1, j-1}: \mathbf{n}_{i+1, j}=\alpha \mathbf{n}_{i+1, j}+(1-\alpha) \mathbf{n}_{i+1, j-1}$. We then calculate $\mathbf{m}=\beta \mathbf{n}_{i+1, j}+(1-\beta) \mathbf{n}_{i, \infty}$ and use that to calculate $A$. It was found that for all other variables apart from $\mathbf{n}$ (and importantly, $n_{e}$ which is derived directly from it) a fully implicit $(\beta=1)$ scheme could be chosen with no significant increase in computational time while providing optimal stability.

In the above, we have included in the definition of $A$ an indication that there are dependencies beyond those explicitly noted. This is because there are frequently other variables which must be included, for example the target thickness, a hot electron population, or an external radiation field; however the major dependencies are often on $T_{e}$ and $n_{e}$. The presence of other materials is taken into account through defining $n_{e}$ as a function of $\mathbf{m}$ with addition of the electron number densities resulting from the other elements, while these other elements will also affect the ion dynamic broadening and the broadening used in the quasistatic ion lineshapes. All of these effects are treated in the same way in this damped, iterative loop. Only $n_{e}$ and $\mathbf{m}$ are treated differently in that another inner iterative loop (3.2.2 to 3.2.2.1) is used in order to allow the convergence in $n_{e}$ to be performed more quickly. No attempt is made to split $A$ as a quadratic in $n_{e}$.

Equation 6 is solved using a 2-step process. Firstly the DTA rates are aggregated. The rate matrix, $A$, is formed on the computer node indexed 0 and is Gauss-Jordan inverted. To give an example the levels $1 s^{2}$, $1 s 2 s$ and $1 s 2 p$ have 7 DTA terms, the rates between which are conglomerated onto the $3 n l$-representation DCA states. The solution is then projected back out on to the DTA vectors by statistical weighting with Boltzmann factors. This vector is then used as the beginning to a parallel Jacobi iterative process, since often the rate matrix is too large to store in RAM on a single node and this allows the entire computational cluster to be utilised.

Jacobi iteration was chosen due to its simplicity and ease of parallelisation, but also has the useful feature, peculiar to this problem, that the first iteration will reproduce coronal equilibrium. Taking the system of $1 s^{2}, 1 s 2 s$ and $1 s 2 p$ at low density as an example, the population is predominantly in the $1 s^{2}$ state and the populations in the $1 s 2 s$ and $1 s 2 p$ terms will be poorly predicted by the projection back with Boltzmann factors due to metastability, particularly in the $1 s 2 s{ }^{3} S_{1}$ case. However, the first iteration of the Jacobi iteration gives the population, $p_{e}$, of any excited state in terms of the ground state, $p_{g}$, and other excited states $p_{e^{\prime}}$ as

$$
p_{e}=\frac{1}{\sum_{e^{\prime}} R_{e \rightarrow e^{\prime}}}\left[R_{g \rightarrow e} p_{g}+\sum_{e^{\prime}} R_{e^{\prime} \rightarrow e} p_{e^{\prime}}\right]
$$

which is, to first order,

$$
p_{e}=\frac{1}{\sum_{e^{\prime}} R_{e \rightarrow e^{\prime}}}\left[R_{g \rightarrow e} p_{g}\right]
$$


and coronal equilibrium is obtained.

At high density, the system is close to LTE and therefore the projection back from the Gauss-Jordan step is close to the correct answer. Thus, we see that the method used here needs one iteration in the case of coronal equilibrium and no iterations in the case of LTE. The ability to obtain the two limits easily suggests that the method will be successful and robust, and indeed the convergence has not been seen to be problematic.

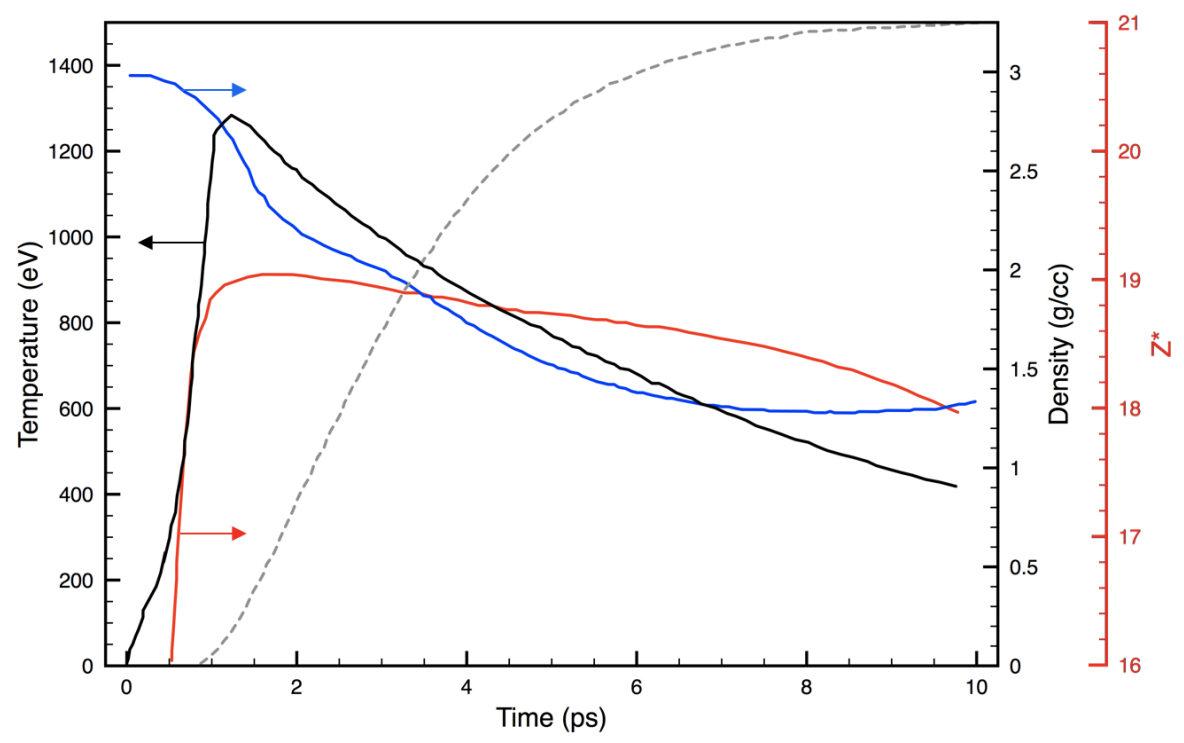

Figure 4: The spatially averaged temperature (black line) and density (blue line) of an initially $0.4 \mu m$ thick Scandium layer predicted by NYM[33][32]. The integrated K-shell emission (black dashed line) and the charge state (red line) calculated by ALICE are also shown.

The capability discussed above proves to be particularly important in the analysis of experiments using short-pulse lasers, an example of this is given in Figure 4. The laser is incident on the front surface of a buried layer target: a thin layer of Scandium sandwiched between two thicker layers of plastic or diamond. The layer is rapidly heated to $\sim 1 \mathrm{keV}$ while the surrounding plastic slows the expansion, meaning that it remains within a factor of a few of solid density. Figure 4 shows how the ionisation change lags behind the temperature change, particularly near the peak in temperature and emission. The spectra produced are sensitive to the time dependence. In addition to the time dependence the damped iterative scheme discussed above is required to obtain consistency and convergence in the radiation field, particularly for the optically thick lines [34]. The criteria for convergence depend on the sum of the absolute value of fractional deviations in all of the calculated quantities, for example $\sum 2\left(\mathbf{n}_{i, j+1}-\mathbf{n}_{i, j}\right) /\left(\mathbf{n}_{i, j+1}+\mathbf{n}_{i, j}\right)$ in the case of the level populations and similar with $I(\nu)_{i, j}$ in the case of the radiation field. This is significantly more stringent than using conditions based on integrated values e.g. $n_{e}$ or the integrated emission. 


\subsection{Line trapping and radiation transport}

ALICE calculates two radiation fields. The first is an averaged radiation field in the target which is used for the solution of the atomic kinetic model, and the second is the radiation field escaping the target at a given angle (corresponding to the observation angle of the spectrometer). For the first case the radiation field is calculated by solving the frequency-resolved radiation transfer equation along a mean chord (given by the Cauchy mean chord theorem) in the plasma. For example, for a slab of plasma where $d$ is the perpendicular thickness the main chord is given by $2 d$. This has been tested against more complex, multizonal 1D simulations and has been seen to give good agreement. For the second case in which the radiation escaping the target is calculated, the radiation transport equation is solved as before but along the line of sight through the plasma. The spectral grid for the radiation field is more detailed in the region of the Kand L-shell of any elements present in the mixture, and the fully detailed radiation field as seen in the output below is used at all stages of the calculation. This means that photopumping both within a material and between different materials can be treated.

We consider Scandium spectra obtained from the ORION laser[32]. Two spectrometers at $15^{\circ}$ and $75^{\circ}$ to the target normal were used. The distance across the Scandium layer along the spectrometer line of sight is a factor of 3 larger in the second case; and this causes a difference between the observed spectra. Figures 5 and 6 show the spectra taken at these two angles. The ratio between the $\alpha$ and $\beta$ line intensities, which in the optically thin limit must be $<A_{\beta} / A_{\alpha} \approx 1 / 4$ (where $A$ are the Einstein A coefficients), is much larger in the second spectrum. This is because at high optical depth the emitted spectrum tends to the source function, and, due to the radiation field affecting the atomic kinetics, the source function tends to a Planckian. The intensities of the Ly- $\alpha$ peaks in the second spectrum are in an almost $1: 1$ ratio, yet in the optically thin limit should have a ratio of $1: 2$ which is determined by the detailed atomic physics. To obtain the ratio observed here, the optical depth at line centre of the more intense line of the doublet must be $\sim 10$, which allows a semi-analytic verification of the thickness of the Scandium layer inferred from the code.

Figures 5 and 6 show that the agreement between the code and the experiment is generally good. The line ratios are well reproduced, the linewidths and shapes are generally accurate, and the agreement at the two different angles from the same simulation are indicative of an underlying accuracy in the model. The time and density histories are extracted from NYM simulations performed at AWE[32]. When ALICE was being developed, it was notable that a lack of inclusion of accurate radiation transport, deficiencies in the lineshape physics, or lack of completeness of the model all led to situations where one of the spectra could be fitted by a change in the radiation hydrodynamic modelling, however the agreement with the other spectrum would always be very poor. Modelling with ALICE suggested that the target thickness was about double that originally inferred during target fabrication which was confirmed by more advanced target metrology.

However, some features are not reproduced as well. The satellites to the He- $\alpha$ line are over-estimated using ALICE, which is possibly due to underestimation of the speed of the target disassembly using a radiation hydrodynamics code. An examination of the sound speed shows that after $\sim 5 p s$ the rarefaction wave from the front surface will have reached the buried layer, and a slightly faster disassembly than that predicted by the radiation hydrodynamics code, will decrease the density and temperature in the layer, decreasing the intensity of those satellites.

Another possible cause of some of the smaller discrepancies in the spectra is the lack of transverse uniformity of the target predicted by $2 \mathrm{D}$ PIC simulations which is not seen in $1 \mathrm{D}$ or $2 \mathrm{D}$ radiation hydrodynamics 


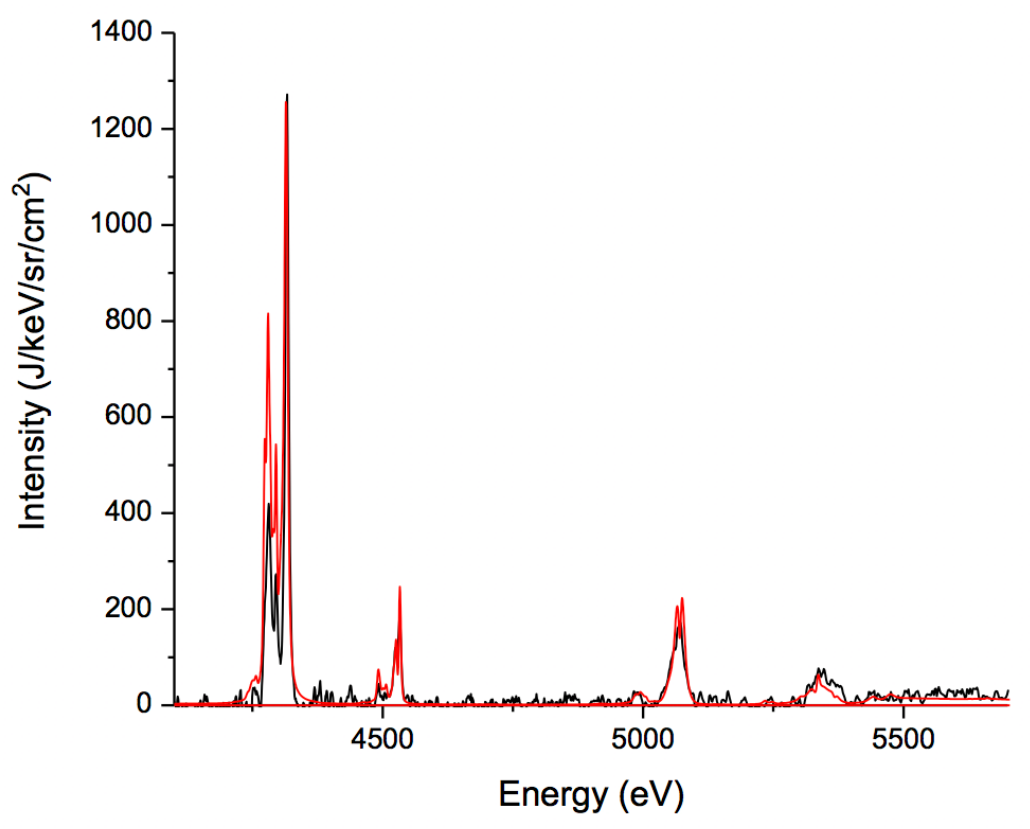

Figure 5: The time-integrated spectrum from the Scandium target which evolved as shown in figure 4 when viewed at $15^{\circ}$ to the target normal. The experimental spectrum is shown in black, the predicted spectrum from ALICE is shown in red.

simulations. The majority of the emission is predicted to occur before transverse perturbations to the temperature of the layer predicted by 2D PIC simulations have been smoothed by thermal diffusion. Such perturbations are predicted to be caused by filamentation of the hot electron and return currents and are consistently seen in 2D PIC simulations of short-pulse laser plasma experiments [35].

\subsection{Completeness of configurations}

Completeness of configurations has become an issue that has received increasing study in non-LTE modelling in recent years. The first issue related to this, involves including a sufficiently large number of configurations that the partition function is converged. This is particularly problematic in situations with large numbers of highly populated excited states, for example near LTE. A second issue arises from the need to include levels of low population that provide important kinetic pathways. At astrophysical densities these issues are successfully mitigated by approximations based on radiative cascades or using resonance contributions to the recombination cross-sections. At higher densities these issues can not be resolved in this way and this need to treat large numbers of excited and doubly excited levels is the major problem in modern non-LTE physics. Here we describe how these levels are included in ALICE. The issue of how many of these high lying levels to include is decided by the pressure ionisation, where the surrounding plasma removes high lying states. 


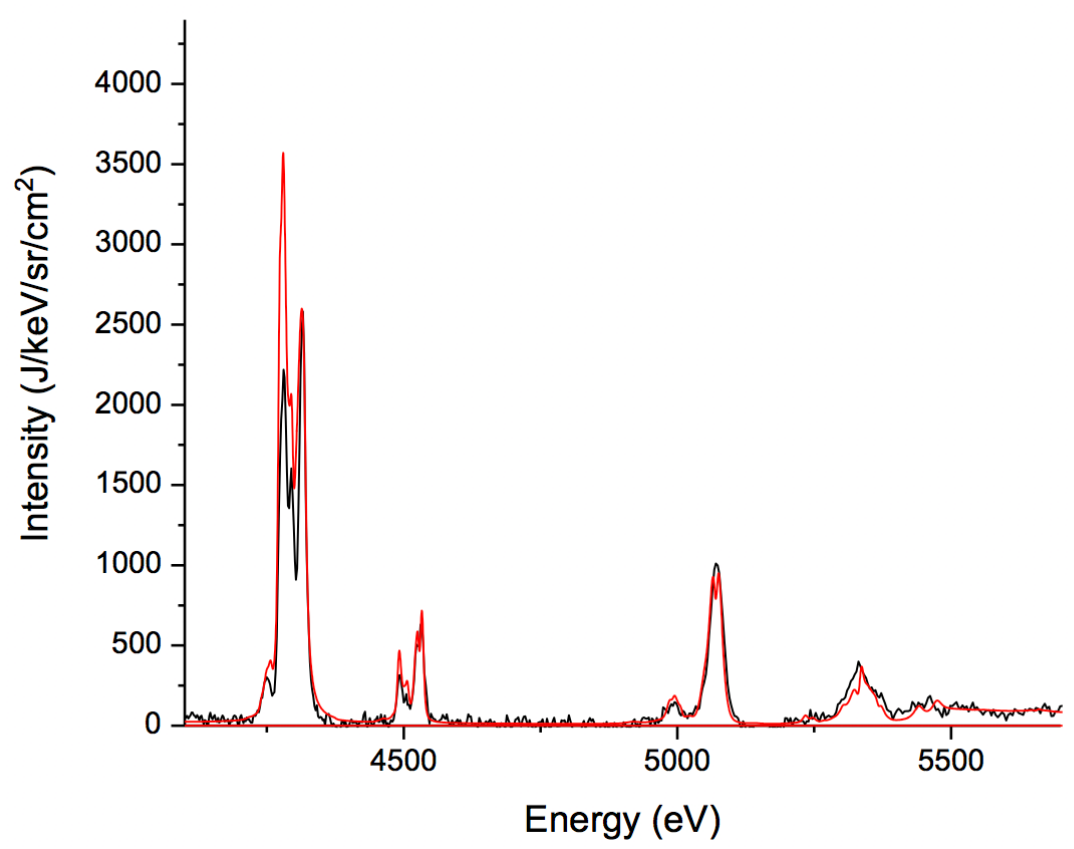

Figure 6: The time-integrated spectrum from the Scandium target which evolved as shown in figure 4 when viewed at $75^{\circ}$ to the target normal. The experimental spectrum is shown in black, the predicted spectrum from ALICE is shown in red.

\subsubsection{The series approach}

Current non-LTE models consider a set of levels $\left\{l_{i}\right\}$ and a set of transitions $\left\{\left(l_{i}, l_{j}\right)\right\}$ between pairs of levels, where each transition is considered entirely separately. This has the advantage of being compact, easily extendible and also follows naturally from LTE codes. While a full Liouville space representation calculation is currently intractable, in ALICE we have attempted to make some steps towards it by dividing the calculation into subsets of transitions, which consist of a series of transitions and their series limit. We have sets of levels and transitions, treated as one object, for example

$$
\left[\left\{1 s^{2}, 1 s 2 s, 1 s 2 p, 1 s 3 s, 1 s 3 p, 1 s 3 d, 1 s \epsilon\right\}\left\{\left(1 s^{2}, 1 s 2 s\right),\left(1 s^{2}, 1 s 2 p\right), \ldots,\left(1 s^{2}, 1 s \epsilon\right)\right\}\right]
$$

or

$$
\text { [\{1s2s, } 1 s 2 p, 1 s 3 s, 1 s 3 p, 1 s 3 d, 1 s \epsilon\}\{(1 s 2 s, 1 s 2 p),(1 s 2 s, 1 s 3 s), \ldots,(1 s 2 s, 1 s \epsilon)\}]
$$

where it will be noted that the sets of levels used to make up these objects are not disjoint, however along with the transitions there is no overlap and each represents a clearly separate physical concept - a series up to a continuum limit - in the first case the ionisation of one of the $1 s$ electrons from $1 s^{2}$ and in the second case the ionisation of the $2 s$ electron from $1 s 2 s$. The properties of the transitions and levels are then considered together, which then allows a natural treatment of the movement of the levels into the 
continuum, and conversely a relatively simple treatment of additional levels which are not ionised. While this may appear cumbersome in a human-readable form, through the localisation of the information into a single data structure it simplifies computations considerably. If one were more concerned about the effects of overlapping lines and the interference between them, transitions grouped by their proximity in energy rather than by series could be optimal, an example of this is found in [36].

Treating problems where levels are removed by continuum lowering is simple in this model, and the representation used here would lend itself to codes seeking to impliment more advanced models of the continuum, for example treating resonances or using the results of models such as PURGATORIO[16].

\subsubsection{Treating Rydberg series}

The representation above makes it easy to extend a series of lines by allowing the code to use a detailed model for the lower series lines which tend to exhibit more term structure and have larger oscillator strengths, but then to switch to a continuation of that series up to the continuum when data is no longer available. This is an alternative to the use of superconfigurations for these high lying levels.

In a given series, the lower level, $n^{\prime}$, is always the same and so its population and energy is known. The upper levels can be found by applying the following changes to the highest known member of the series, the $n^{\prime} \rightarrow n$ transition, where $n^{\prime}$ and $n$ are principal quantum numbers with energy $E_{n}$, for some other transition $n^{\prime} \rightarrow m$ with energy $E_{m}$ with $m>n$. We define the energies to be relative to the unperturbed continuum. First we consider the energies, which in a screened hydrogen approximation are given by

$$
E_{m}=E_{n}\left(\frac{n}{m}\right)^{2}
$$

Since the $Z^{* 2}$ of the screened hydrogen model can be found as $E_{n} n^{2}$ and then used in the calculation of $E_{m}$. The oscillator strength is given by

$$
f_{n^{\prime} \rightarrow m}=\left(\frac{E_{n} n}{E_{m} m}\right)^{3} f_{n^{\prime} \rightarrow n}
$$

which is Unsold's formula [30], and which should approximately preserve the sum rule as the continuum is lowered (this can easily be ensured exactly in the series representation used here). The width of the line is given by

$$
w_{n^{\prime} \rightarrow m}=\left(\frac{m}{n}\right)^{4} w_{n}
$$

where the scaling is with the fourth power from the $\mathbf{r}^{2}$ matrix element in the line width. If the system is in LTE, the population of the upper level, $p_{m}$, can be found either by using a Boltzmann factor from the population of the level below it, $p_{n}$, or by a Saha-Boltzmann factor from the population of the level it ionises to, $p_{+}$, with both methods necessarily giving the same result. However if the system is not in LTE these methods do not produce the same result, and so it is natural to consider a method which obtains the correct answer in LTE and merges between the two methods of calculating the population, $p_{m}$, depending on which would be expected to be the most valid. We choose an expression of the form

$$
p_{m}=\left(1+a_{1} \chi_{m}^{a_{2}}\right)^{ \pm 1} S\left(n_{e}, T_{e}, \chi_{m}\right) p_{+}
$$

where $S\left(n_{e}, T_{e}, \chi_{m}\right)=n_{e} \Lambda\left(T_{e}\right) e^{-\chi_{m} / T_{e}}$ is the Saha-Boltzmann factor, $\chi_{m}$ is the ionisation energy of the state taking into account the pressure ionisation term (i.e. if the level were about to be ionised $\chi_{m}=0$ and 
when the level exists $\chi_{m}<0$ ), and $\Lambda$ is the thermal be Broglie wavelength. \pm 1 is used as the exponent to allow $p_{m}$ to be either less than or greater than $p_{+}$, and provides a simple function with the desired property of switching from $p_{m}$ - to $p_{+}$-dominated behaviour when $a_{1} \chi_{m}^{a_{2}} \sim 1$. The Griem condition [51] is derived by considering the scalings of the collisional and radiative rates with energy changes. This condition, and further discussions comparing different sets of rates, conclude that the magnitude of deviation of a level from LTE is determined by a parameter $a_{1} \chi_{m}^{a_{2}}$, where $a_{2}$ is a value in the range $3-4$. $a_{1}$ may then be determined for the system considered here by solving

$$
p_{n}=\left(1+a_{1} \chi_{n}^{a_{2}}\right)^{ \pm 1} S\left(n_{e}, T_{e}, \chi_{n}\right) p_{+}
$$

where $p_{n}$ and $p_{+}$are known, being the population of the highest level in the atomic structure model and the population of the ionised state respectively. We are introducing a deviation from the normal Boltzmann factor to correct for departures from LTE - if we are in LTE, $a_{1}=0$ and so $p_{m}=S\left(n_{e}, T_{e}, \chi_{m}\right) p_{+}=p_{n} B\left(T_{e}\right)$ where $B\left(T_{e}\right)$ is the Boltzmann factor $B\left(T_{e}\right)=\exp \left(\left(E_{m}-E_{n}\right) / T_{e}\right)$. In non-LTE, we have $p_{m} \rightarrow S\left(n_{e}, T_{e}, \chi_{m}\right)^{ \pm 1} p_{+}$ as $m \rightarrow M$, its largest value (which is finite due to pressure ionisation), since $\chi_{m} \rightarrow 0$, as expected. Clearly, more sophisticated expressions could take the place of $\chi_{m}^{a_{2}}$ if this were considered necessary. The Chlorine case below is in LTE, and $a_{1}=0$, however in the Scandium case the He- $\delta$ line $(n=1 \rightarrow 5)$ at $5450-5500 \mathrm{eV}$ is included in using this method with $a_{1} \neq 0$.

\subsubsection{Supplementing the edges}

Finally, extra ionisation edges from the Rydberg levels must be added. This uses the ionisation $n \rightarrow \epsilon$ for the largest available $n$ and hydrogenic scaling to simulate the effect of multiple $m \rightarrow \epsilon$ transitions. The scalings are as $(m / n)^{-5}$ in strength and $(m / n)^{2}$ in degeneracy. In order to account for the $m \rightarrow m+k, k>0$ transitions, the photoionisation edges are extended downwards in energy to the energy of the $m \rightarrow m+1$ transition.

\subsubsection{Satellite lines}

The kinetics model will also attempt to include satellite lines not included in the atomic data by using a formula for the population of the upper and lower levels of the satellite lines analogous to that decribed for Rydberg series above. It is primarily designed to help to include Rydberg satellites. We look at a primary transition $a \rightarrow b$, and now add an electron anl $\rightarrow b n l$ : In the limit of the $n l$ electron being uncoupled the line will look exactly the same as the primary line but with a different population of the upper and lower states. As the electron becomes increasingly coupled to the other electrons through the direct and exchange interactions, i.e. as $n$ decreases, the satellite line will shift to lower energies, there will be a contribution to the broadening, and the term structure will change. We approximate this by neglecting the broadening and the change in term structure. The broadening is neglected as a simple approximation to the interference effects described in [37], and the term structure is neglected since if the term structure is important, the satellites should be included in the detailed model.

Satellite lines are often important in high density and temperature plasmas such as those ALICE is designed to model. The magnitude of the satellite lines can be estimated as $\frac{g_{s}}{g_{l}} n_{e} \Lambda^{3}$ times that of the primary transition, where $g_{l}$ is the degeneracy of upper level of the transition and $g_{s}$ that of the satellites. 
This can be a significant fraction of the strength of the primary line. In Scandium, at half of its solid density and a temperature of $1.2 \mathrm{keV}$ the satellites from the $n=3-5$ levels can add $20 \%$ to the strength of a line; at lower temperatures, for K-shell Aluminium, the differences are even more pronounced. When ALICE is used, the number of configurations needed may preclude putting all of the satellite lines to a transition into the atomic kinetics model and in that situation the satellite lines can be included in an approximate way using the scheme described in this section. This has two purposes: Firstly in situations where the approximations are justified, it provides a way to include the many satellites on the low energy wing of stronger lines with good fidelity but with a small increase in computational time. Secondly, by switching on and off this method of including satellites, it can be used to highlight areas where the set of configurations used in the atomic kinetics model should be extended to include more satellite lines.

When new levels are included which are not in the underlying set of levels, higher members of a Rydberg series, for example, the populations of those new levels are summed. The total ion population is renormalised and the charge state recalculated, ensuring conservation of number and charge when these new levels are included.

\subsection{Chlorine spectra $T_{e}=100 \mathrm{eV}, \rho=0.005 \mathrm{~g} / \mathrm{cc}$}

Having discussed the methods used in ALICE to ensure completeness of configurations in the previous Section, we consider the absorption spectrum of Chlorine in LTE at $T=100 \mathrm{eV}$ and $\rho=0.005 \mathrm{~g} / \mathrm{cc}$. This will be used to demonstrate the method of including Rydberg series, and the ability to reproduce to a good accuracy, the results from other well benchmarked codes.

The Rosseland opacity, $\kappa_{R}$, is defined by $\kappa_{R}^{-1}=\int \kappa(\nu)^{-1} u(T, \nu) d \nu / \int u(T, \nu) d \nu$ with $u(T, \nu)=\partial B_{T}(\nu) / \partial T$. $u(T, \nu)$ for $T=100 \mathrm{eV}$, peaks at $\sim 380 \mathrm{eV}$. $\kappa_{R}$ is therefore primarily determined by the spectrum in this region which is a 'window', a region of low opacity, bounded on the high energy side by the low energy (and therefore low charge state) $n=2 \rightarrow 3$ transitions at $300-400 \mathrm{eV}$ and with its depth determined by the $n=3 \rightarrow n$ transitions running up to the M-shell ionisation edge at $\sim 400 \mathrm{eV}$ (Figure 7 , red line). This makes inclusion of the high Rydberg states crucial to get an accurate value of $\kappa_{R}$. It is important that ALICE can produce reasonable agreement with other codes so that comparisons can be made and so the effect of non-LTE physics on Rosseland opacities can be determined. Here we demonstrate good agreement between ALICE and the online tabulations of the LANL opacity codes [40] and also compare with IMP, an average atom LTE opacity model [38].

Figure 7 shows the frequency resolved spectra with (red) and without (black) including the extra edges and extra Rydberg series lines calculated by ALICE. The filling in of the window can be clearly seen, and while the Planck opacity shows a $<10 \%$ difference between the two spectra, the Rosseland opacity calculated by ALICE without the extra Rydberg levels and edges included is too small by a factor of 2. In Figure 8 where we compare against the LANL codes in the same conditions some differences exist, in the line broadening for example, and the treatment at low frequency; however the overall shape of the frequency dependent opacity is very similar. This leads to a similarity in the Rosseland mean opacities (Table 2), with a difference of $\sim 5 \%$ between the detailed codes, along with differences of $\sim 0.1$ in the charge state, as expected. We also compare the values for the Rosseland opacity and charge state against IMP[38], an average atom model. The agreement between ALICE and IMP is fair. It must be emphasised however that the computational time needed to run IMP is very much lower than that needed by ALICE (by about 2 orders of magnitude) and 
this example shows the value of more detailed codes for performing spot-checks on less detailed codes which are however more amenable to the production of large scale tabulated data. While modern computational facilities make tabulation in $\rho$ and $T$ practical with detailed codes for low to mid-Z elements, for high-Z elements or if higher dimensional tables, including mixtures of materials for example, are required, codes such as IMP or CASSANDRA[39] are still generally used.

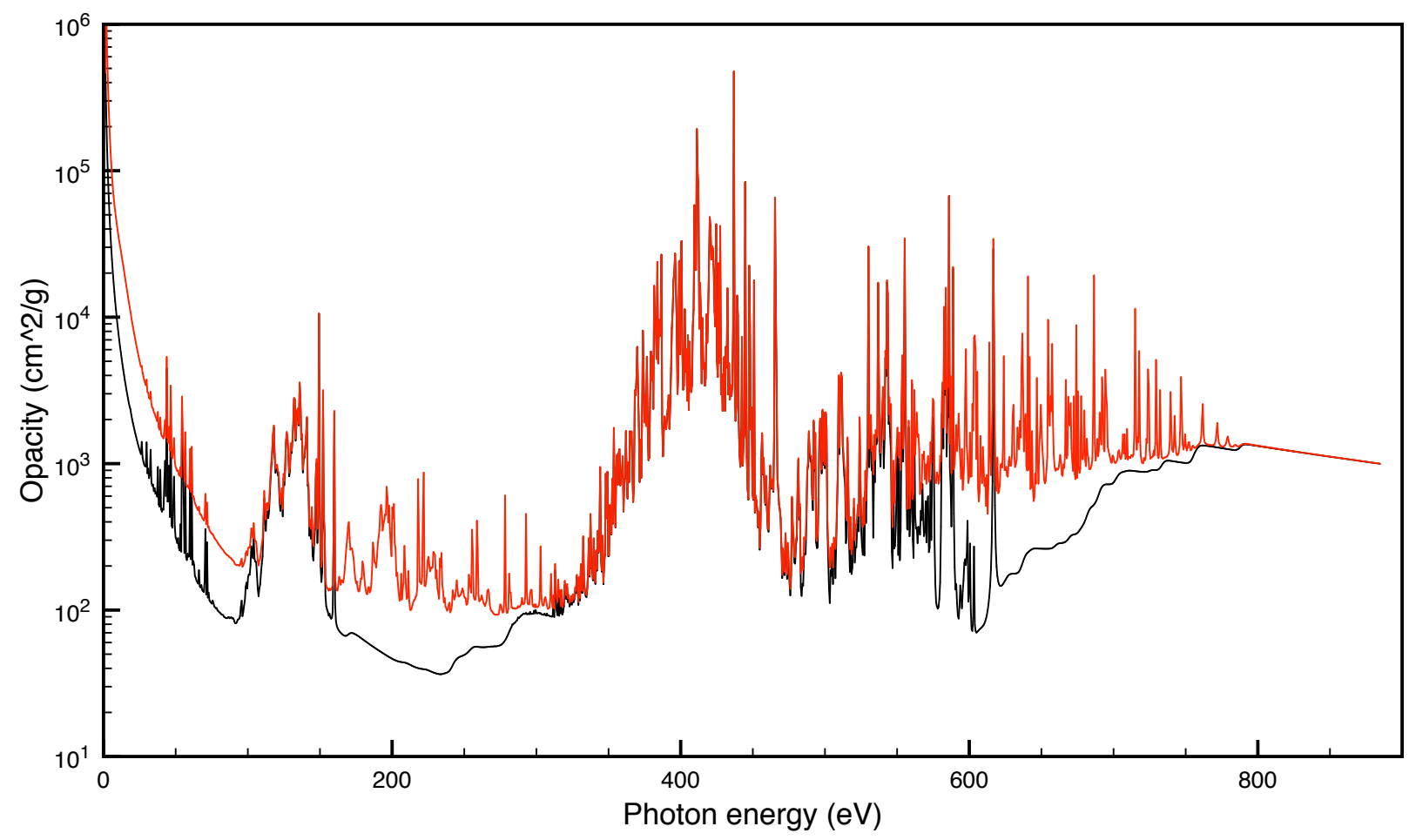

Figure 7: A comparison of the frequency resolved LTE opacities of Chlorine calculated by ALICE. The density if $0.005 \mathrm{~g} / \mathrm{cc}$ and the temperature is $100 \mathrm{eV}$. The curves show the result with (red), and without (black), the inclusion of the extra Rydberg levels and edges.

\subsection{Germanium spectra - $300-700 \mathrm{eV}, 1 \mathrm{~g} / \mathrm{cc}$}

Here we consider Germanium and compare to experimental results from AWE, again from a buried layer target[41], to demonstrate the ability of ALICE to combine DTA and DCA representations in a single calculation and thereby to treat non-LTE open L-shell problems without incurring a large increase in computational time compared to the simpler Scandium K-shell problem above. These experiments have previously been modelled using DTA and average atom LTE codes and in steady state using FLYCHK, a non-LTE model, which uses a UTA formalism for the majority of the spectrum [41].

As the L-shell is opened the number of possible configurations to be included in the model increases rapidly, as is also the case but to a greater extent for the $\mathrm{M}$ - and $\mathrm{N}$-shells. It is well known that both single 


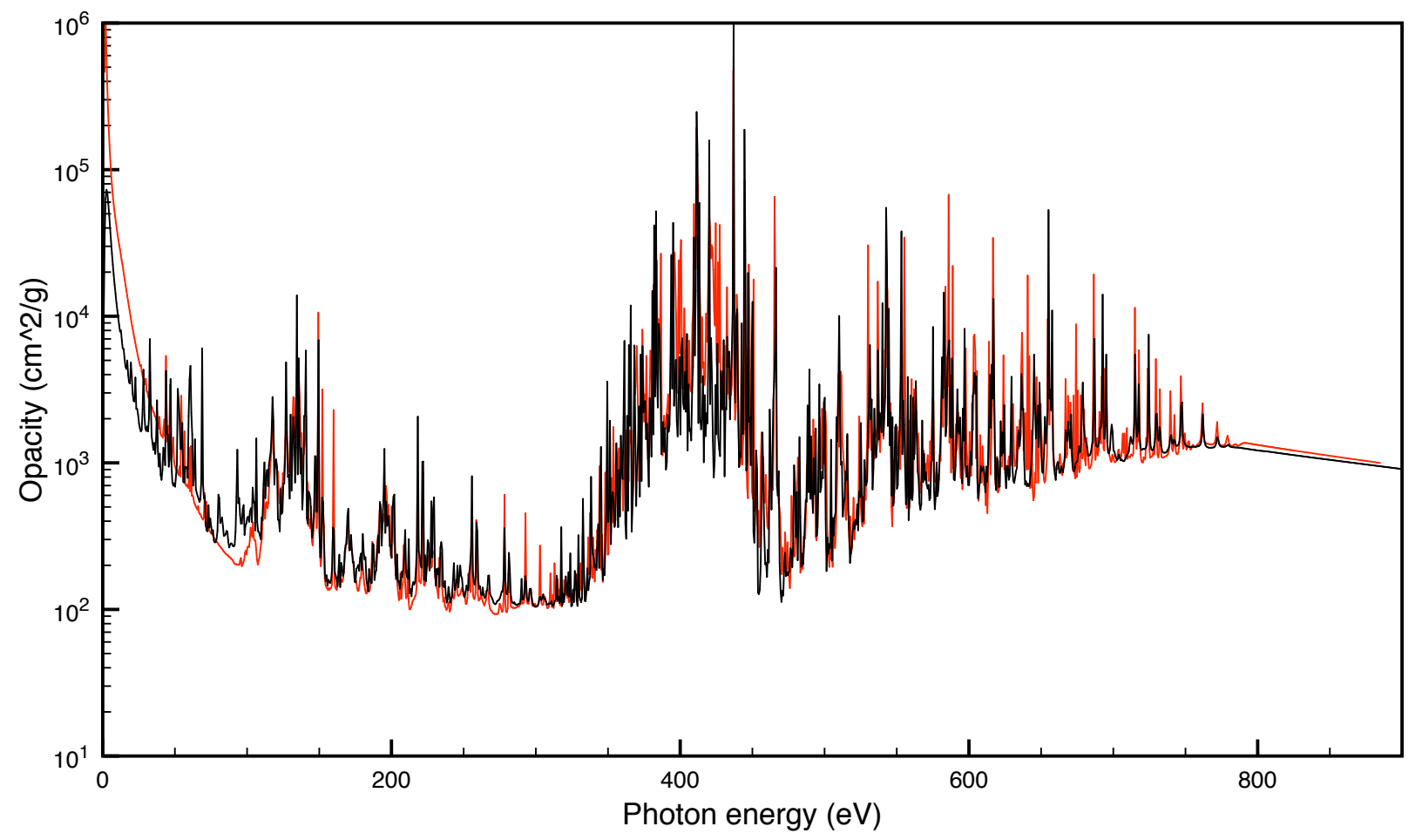

Figure 8: A comparison of the frequency resolved LTE opacity of Chlorine calculated by ALICE (red) and the LANL opacity codes [40] (black). The density if $0.005 \mathrm{~g} / \mathrm{cc}$ and the temperature is $100 \mathrm{eV}$.

(to obtain the spectral lines) and double (to obtain the autoionisation rates) excitations from the ground state must be included and inclusion of doubly excited states leads to a very large number of levels in the atomic model. This is particularly problematic since all the rates between those levels must be calculated, unlike if the assumption of LTE and statistical population of the levels is made.

The autoionising states in this case are included using the hybrid DTA-DCA scheme. The configurations are listed in the input file, with ground states and single excitations treated in a DTA formalism and the doubly excited states included in a DCA formalism. Both are then included in the complete model. Including doubly excited states causes two differences to the spectrum. The first is a filling in of the dips between the strong lines, caused by the presence of the autoionising satellite lines and the second is a small change in ionisation state due to the inclusion of autoionisation. In Figure 10 we show calculations of the spectrum of Germanium at $900 \mathrm{eV}$ and $1.5 \mathrm{~g} / \mathrm{cc}$ with solely single and single and double excitations included in the model to demonstrate these effects.

The Germanium case, as has been noted in previous papers[41], is a sensitive test of the accuracy of the energy levels and therefore transition energies calculated by the atomic physics code. The position of the transitions can be accurately obtained from the spectra, particularly in the higher energy $(\gtrsim 1700 \mathrm{eV})$ Lithium- and Beryllium- like lines where the simpler term structure leads to the lines being well-separated 


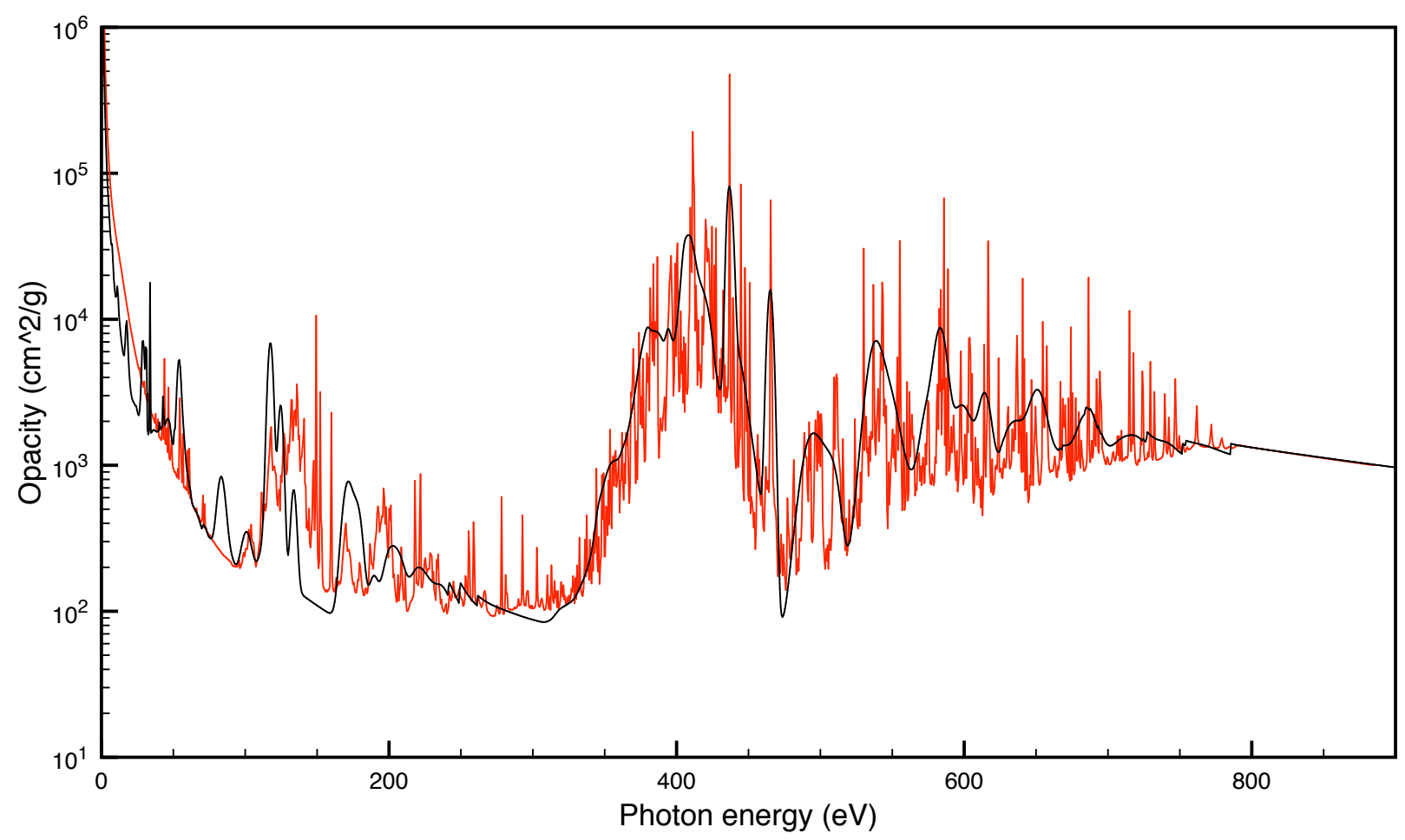

Figure 9: A comparison of the frequency resolved LTE opacity of Chlorine calculated by ALICE (red) and IMP [38] (black). The density if $0.005 \mathrm{~g} / \mathrm{cc}$ and the temperature is $100 \mathrm{eV}$.

and readily identifiable. The accuracy of the energy levels in ALICE is similar to those in other modern DTA LTE opacity codes (e.g. [42] Figure 4), and to reiterate that paper's conclusion, shows the continuing value of experimental data even in these relatively simple cases. The discrepancy of $+2.5 \mathrm{eV}$ in the position of the strong $1 s^{2} 2 p_{1 / 2} \rightarrow 1 s^{2} 3 d_{3 / 2}$ line at $1765 \mathrm{eV}$ is a deficiency shared by this code, LANL[43], and NIST data; and there are also other discrepancies, which point to the usefulness of further investigation of line broadening mechanisms and the inclusion of configuration interaction as well as into the effect of time dependence on the problem. The spectrum from ALICE in Figure 10 has not been scaled in intensity and so ALICE reproduces the absolute emissivity measured in the experiment well.

\subsection{Line broadening}

Line broadening is very important in producing results which are qualitatively consistent with experiment and is crucial if quantitative comparisons are to be made. In this section we will describe the line broadening mechanisms implemented in ALICE.

As is standard in line broadening theory, we use the observation that generally the electron timescales are significantly faster than the ion timescales and so separate the contibutions from the two species. The fluctuations related to the electrons lead to the elastic electron broadening contribution and (through the 


$\begin{array}{llll}\text { Density }(\mathrm{g} / \mathrm{cc}) & \text { ALICE } & \text { LANL } & \text { IMP } \\ 0.001 & 52.19 & 53.27 & 67.08 \\ & 14.44 & 14.39 & 14.67 \\ 0.005 & 359.9 & 378.3 & 358.9 \\ & 13.58 & 13.53 & 14.02 \\ 0.01 & 687.6 & 719.1 & 686.8 \\ & 13.10 & 12.95 & 13.60\end{array}$

Table 2: A comparison of the Rosseland mean opacities from ALICE, LANL [40] and IMP [38]. The opacities are given in $\mathrm{cm}^{2} / \mathrm{g}$ and the values of $Z^{*}$ are shown in italics.

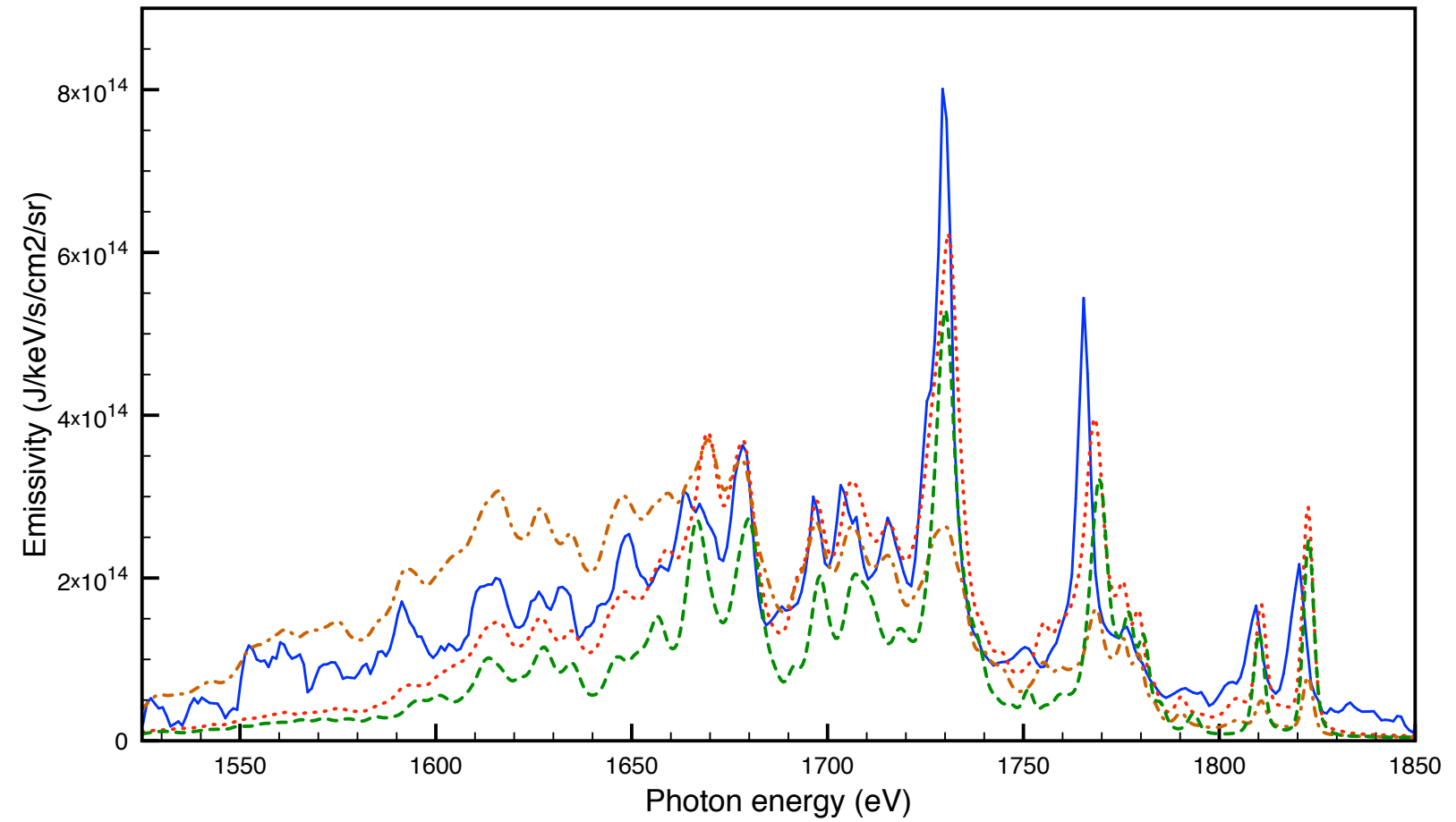

Figure 10: Spectrum of Germanium from a short pulse buried layer taget experiment [41] (blue solid line). Theoretical spectrum produced by ALICE including double excitations (red dotted line) and single excitation (green dashed line) in a DCA representation at a temperature of $900 \mathrm{eV}$ and density $1.5 \mathrm{~g} / \mathrm{cc}$. An instrument function of $2.5 \mathrm{eV}$ FWHM is convolved with the output. The two excitations ALICE spectrum for $700 \mathrm{eV}$ is also included for comparison (brown dot-dashed line). Time-dependently there will be contributions from a range of temperatures, the lower temperatures contributing to the lower energy members of the array in the $\lesssim 1650 \mathrm{eV}$ region as shown in this graph. 
atomic kinetic model) to a significant fraction of the inelastic broadening; these are both treated in the impact approximation. The ion broadening is treated separately - as a first approximation the ions are not moving and so the quasistatic approximation is used which is then corrected using a fluctuation model..

\subsubsection{Inelastic broadening}

Broadening due to inelastic effects - collisional and radiative transitions - is included in the lifetime broadening of the levels, which is derived from the diagonal rate matrix elements. One problem is that as a level moves towards the continuum, analytic approximations to the collisional ionisation rates can become very large; or in the case of a collisional excitation at high density, the rate of excitation, $R$, can be equivalent through $E=h R$ to an energy, $E$, larger than the energy difference, $\Delta E$, between the levels. In ALICE the largest of the excitation and de-excitation rates is bounded above by $\Delta E / h$ (and the other decreased by the same fraction so as to maintain detailed balance), a manifestation of both the uncertainty principle (the levels cannot be distinguished). And also a breakdown of Fermi's Golden Rule on which the rate calculations are based. Regarding the atomic kinetics, the collisional rates between these levels are then so large that they are in thermodynamic equilibrium, however as regards the spectrum, cases like this should be treated using Rabi oscillation-type arguments and the lineshapes should show the effect of dynamic broadening; in particular motional narrowing will eventually become apparent. To the authors' knowledge this has not been investigated.

\subsubsection{Elastic broadening}

Elastic broadening due to the electrons is included using the formalism of Dufty [44] and we use the approximations for his $G$ function discussed by Lee [45].

\subsubsection{Ion dynamic broadening}

Ion dynamic broadening - the fact that the ion microfield fluctuates between different values with a finite and non-negligible frequency in hot plasmas - is included using the Frequency Fluctuation Model [46] with the clarifications from [47]. The fluctuation rate is given by [48], where the prefactor to the basic $n_{i}^{1 / 3} T_{i}^{1 / 2} \mu^{-1 / 2}$ scaling, $1.92 \times 10^{-10} \mathrm{eV}^{-1 / 2} \mathrm{~ms}^{-1}$ used in ALICE, is consistent to within $7 \%$ to either the computational work in [46] or the $\nu$ derived from [48] using the fluctuation rate at the maximum of the Holtzmark distribution. ALICE is often used to treat mixtures of materials. Considering the origin of the terms in [48], which treats stellar motion, and making the connection between gravitational mass and charge, and inertial mass and atomic mass, we redefine $T_{i} / \mu$ in the above equation as

$$
\frac{\sum_{j} f_{j} \frac{T_{j} Z_{j}^{1 / 2}}{\mu_{j}}}{\left(\sum_{j} f_{j} Z_{j}^{3 / 2}\right)^{1 / 3}}
$$

for a number of species with temperatures $T_{j}$, masses $\mu_{j}$ and number fractions $f_{j}$. This reproduces the result for a single species with a single $T_{1}=T_{i}, \mu_{1}=\mu$ and $f_{1}=1$. 


\subsection{Ion broadening and the quadrupole effect}

For plasmas at these densities quasistatic ion broadening is an important broadening mechanism, both from the point of view of radiation transport and for diagnostics.

We use a simple quasistatic broadening model - the electron impact widths are given by the same model as used in the usual lines, and the quasistatic shapes are calculated in the usual way [45] in the Liouville formalism. The plasma has two components - the radiating species and a perturbing species, since we use the standard APEX model[49]. We use a simple average for the charge on the perturber when multiple species are present, which, for the relatively weakly coupled plasmas we are considering is deemed sufficient.

While the effects described above are those usually included in a non-LTE model, the widths, particularly of the Ly- $\gamma$ and He- $\gamma$ lines are significantly larger experimentally than would be expected from these standard broadening models. ALICE has the capability to include quasistatic ion quadrupole effects in the broadening calculation, on the same footing as ion dipole effects and therefore again in line with the code as described above. The distribution of the dipole and quadrupole fields required, $Q\left(E, q_{z z}, q_{x x}, q_{y y}, q_{x z}, q_{x y}, q_{y z}\right)$ in the electric field in the $z$-direction and the 6 independent components of the quadrupole tensor (which replaces the dipole distribution $Q(E)$ from APEX usually used) is found using an in-line Monte-Carlo simulation of the plasma structure. Full details of the implementation of the quadrupole broadening along with further examples of its use in Aluminium plasmas are given in [50]. The use of this broadening model below provides a significantly different, though not conclusively better, fit to the He- $\gamma$ line shape in the case of these Scandium spectra and is also seen to rectify significantly larger problems with the fitting of Aluminium spectra.

The Scandium experiment discussed at the beginning of the section is shown below. The instrument function in this experiment can be bounded by considering the resolution of the Ly- $\alpha$ doublet and of the features to the low energy side of the He- $\alpha$ line, and we can therefore compare the spectrum of the He- $\gamma$ line with and without the quadrupole broadening model to investigate this effect, while the goodness of fit to the rest of spectrum ensures that the conditions are known with the accuracy needed. We propose that a quadrupole broadening model is required to remedy the discrepancy seen, specifically that the sharply peaked feature in the dipole calculation at $5335 \mathrm{eV}$ should be clearly visible with this spectrometer in these conditions, while the overall 'triangular' shape of the line is fitted much better by the model including quadrupole effects (see figure 11). It is however acknowledged that other effects discussed above or explicitly ignored in ALICE, particularly spatial non-uniformity, could be responsible in some way for these effects.

\section{Conclusions}

In this paper we have presented the physical models used in the non-LTE plasma atomic physics, kinetics and lineshape package ALICE and demonstrated the need for those models in conditions of interest by comparisons of ALICE's output both against that of other codes and against experimental data. We have shown a capability to model in LTE at low density with the approximate inclusion of Rydberg states, an open

L-shell capability at solid density, the modelling of time dependent non-LTE problems in materials where the radiation transport in non-analytic lineshapes is important and the need to include higher order multipole quasistatic ion broadening to reproduce experimentally observed spectra from solid density plasmas. 

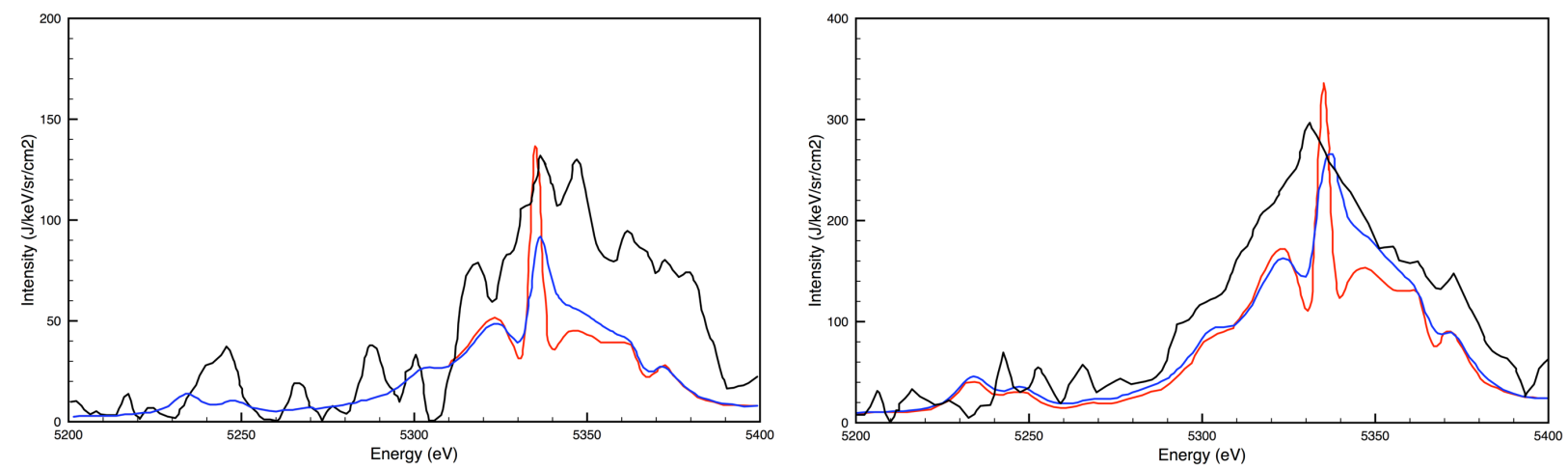

Figure 11: The time-integrated spectra from the Sc target as shown in figures 5 and 6 showing in detail the He- $\gamma$ profiles. The left panel corresponds to $15^{\circ}$ and the right panel $75^{\circ}$ to target normal. The black curve is the experimental data, the red curve is the simulated spectrum from ALICE. In no case is there very good agreement between theory and experiment. However, the overall triangular shape of the feature is better reproduced by the quadrupole model (blue).

\section{Acknowledgements}

The authors would like to thank D. Hoarty and team for providing both previously unpublished and electronic versions of previously published data for comparison in this paper. EGH was supported by AWE, Aldermaston, and would like to thank the reviewer for their helpful comments.

\section{References}

[1] D. H. Sampson, H. L. Zhang, C. J. Fontes Physics Reports 477, 111 (2009)

[2] M. Klapisch, M. Busquet, and A. Bar-Shalom, AIP Conference Proceedings, 926, 206 (2007)

[3] Gu M.F. Can.J. Phys., 86675 (2008)

[4] S J Rose, J. Phys. B: At. Mol. and Opt. Phys. 312129 (1998)

[5] L. Upcratt, Private communication, AWE, Aldermaston (2016)

[6] J.W.O. Harris, L.M. Upcraft, D.J. Hoarty, B.J.B. Crowley, C.R.D. Brown, S.F. James, High Energy Density Physics, 6, 95, (2010)

[7] S. J. Rose, J. Phys B: At. Mol. and Opt. Phys. 25, 1667 (1992)

[8] H.-K Chung et al, High Energy Density Physics, 1, 3 (2005)

[9] S.B. Hansen et al, High Energy Density Physics, 3, 109 (2007) 
[10] H.A. Scott, JQSRT 71, 689 (2001)

[11] Ferri S. et al., Atoms 2014, 2, 299, (2014)

[12] FLYCHK manual, http://nlte.nist.gov/FLY/Doc/Manual_FLYCHK_Nov08.pdf

[13] E. G. Hill JQSRT, 140, 1 (2014)

[14] E. G. Hill, JQSRT,147,71 (2014)

[15] R. D. Cowan, Theory of Atomic Structure and Spectra

[16] B. Wilson, V. Sonnad, P. Sterne, W. Isaacs JQSRT, 99 (2006), p. 658

[17] K.G. Dyall, I. P. Grant, C.T. Johnson, F. A. Parpia, E. P. Plummer, Comput. Phys. Commun., 55, 424, (1989) (for the version discussed in [20] and here)

[18] W. Eissner, M. E. Galavis, C. Mendoza, C. J. Zeippen, ApJS, 137, 165 (1999) W. Eissner, M. Jones, H. Nussbaumer, Comput. Phys. Commun., 8, 271 (1974)

[19] C. Froese-Fischer, Comput. Phys. Commun., 64, 369 (1991) D. C. Griffin, N. R. Badnell, M. S. Pindzola, J. A. Shaw, J. Phys. B, 32, 2139 (1999)

[20] K. M. Aggarwal, F. P. Keenan, and A. Z. Msezane, A and A 410, 349 (2003)

[21] Bar-Shalom, Klapisch and Oreg Phys. Rev. A., 38, 4 (1988)

[22] J. C. Slater, Quantum Theory of Atomic Structure. New York: McGraw-Hill. - Volume 1 (1960)

[23] Y.-K. Kim, Phys. Rev. A., 64, 032713 (2001)

[24] L. M. Upcraft, High Energy Density Physics, 6,332 (2010)

[25] H.-K. Chung et al, High Energy Density Physics, 3, 342 (2007)

[26] H. Chen et al. Ap.J, 646, 653, (2006)

[27] C. D. Lin et al. Phys. Rev. A., 15,1046 (1977)

[28] Inokuti, Reviews of Modern Physics, 43, 3 (1971)

[29] C. J. Bostock et al., Phys. Rev. A. 88, 012711 (2013)

[30] H. A. Bethe and E. E. Salpeter, Quantum Mechanics of One- and Two-Electron Atom,

[31] https://www.imperial.ac.uk/admin-services/ict/self-service/research-support/hpc/

[32] D. J. Hoarty; Private communication

[33] P. D. Roberts, S. J. Rose, P. C. Thompson, R. J. Wright, J. Phys. D, 13, 1957 (1980) 
[34] H.A. Scott andS.B. Hansen, High Energy Density Physics, 6, 39 (2010)

[35] Mark Sherlock, private communication

[36] R. C. Mancini, C. A. Iglesias, S. Ferri, A. Calisti, R. Florido, High Energy Density Physics, 9, 731 (2013)

[37] C. A. Iglesias, High Energy Density Physics, 6, 318 (2010)

[38] S. J. Rose, private communication

[39] B.J.B. Crowley and J.W. Harris, Journal of Quantitative Spectroscopy and Radiative Transfer, 71, I257 (2001)

[40] The online LANL opacity codes - http://aphysics2.lanl.gov/opacity/lanl/

[41] J.W.O. Harris, L.M. Upcraft, D.J. Hoarty, B.J.B. Crowley, C.R.D. Brown, S.F. James, High Energy Density Physics, 6, 95 (2010) D. J. Hoarty et al. Journal of Physics: Conference Series 244012002 (2010)

[42] M. Jeffery, L.M. Upcraft, J.W.O. Harris, D.J. Hoarty High Energy Density Physics, 9, 642 (2013)

[43] The online LANL atomic physics codes aphysics2.lanl.gov/cgi-bin/ION/runlanl08d.pl

[44] James W. Dufty, Phys. Rev. 187, 305 (1969)

[45] R.W. Lee, Proceeds of spectroscopy workshop (1982)

[46] B.Talin et al. Phys. Rev. A, 51, 3 (1995)

[47] C. A. Iglesias, High Energy Density Physics, 9,209 (2013)

[48] S. Chandrasekhar, J. von Neumann, Astrophysical Journal, 95, 489 (1942)

[49] C. A. Iglesias, J. L. Lebowitz, and D. MacGowan, Phys. Rev. A 28, 1667 (1983)

[50] E. G. Hill, to be published

[51] H.R. Griem, Physical Review 131, 31 (1963) 